\title{
Dynamic Reversible Evolution of Wrinkles on Floating Polymer Films under Magnetic Control
}

\author{
Chenchen Jiang ${ }^{1}$, Xue Han ${ }^{2, *}$, Juanjuan Wang ${ }^{2}$, Lele Li ${ }^{1}$, Enping Liu ${ }^{1}$ and Conghua Lu ${ }^{1,2, *}$ \\ 1 School of Materials Science and Engineering, Tianjin University, Tianjin 300072, China; \\ jcc1126@tju.edu.cn (C.J.); lelelee86@163.com (L.L.); epliu@tju.edu.cn (E.L.) \\ 2 School of Materials Science and Engineering, Tianjin Key Laboratory of Building Green Functional Materials, \\ Tianjin Chengjian University, Tianjin 300384, China; wangjj@tcu.edu.cn \\ * Correspondence: hanxue@tcu.edu.cn (X.H.); chlu@tju.edu.cn (C.L.)
}

Citation: Jiang, C.; Han, X.; Wang, J.; Li, L.; Liu, E.; Lu, C. Dynamic Reversible Evolution of Wrinkles on Floating Polymer Films under Magnetic Control. Coatings 2021, 11, 494. https:// doi.org/10.3390/coatings11050494

Academic Editor: Aurora Diana Rata

Received: 25 March 2021

Accepted: 20 April 2021

Published: 23 April 2021

Publisher's Note: MDPI stays neutral with regard to jurisdictional claims in published maps and institutional affiliations.

Copyright: (c) 2021 by the authors. Licensee MDPI, Basel, Switzerland This article is an open access article distributed under the terms and conditions of the Creative Commons Attribution (CC BY) license (https:// creativecommons.org/licenses/by/ $4.0 /)$.
Abstract: In this paper, we present a simple and versatile method to dynamically and reversibly tailor surface wrinkles on a floating polymer film by combining a magnetic droplet and neodymium magnet. The magnetic force from the attraction of the neodymium magnet to the magnetic droplet is the main reason for surface instabilities of floating polymer films, which can induce radial stress in the radial direction, and further, compressive stress in the circumferential direction. This compressive stress can trigger not only floating film wrinkling but also a wrinkle-fold transition. Surface morphologies on the floating polymer film have been systematically studied, by varying the distance between the magnetic droplet and neodymium magnet, polymer film thickness, and magnetic droplet volume. With the decrease in the distance between a magnetic droplet and a neodymium magnet, the decrease in polymer film thickness, and the increase in the magnetic droplet volume, the wrinkle numbers increase and even a wrinkle-fold transition happens. Additionally, the coupling effect of multiple magnetic droplets on the floating film has also been used to achieve novel surface wrinkle patterns, which greatly widens the applications of surface wrinkling.

Keywords: surface wrinkling; floating polymer films; dynamical reversible wrinkles; magnetic field

\section{Introduction}

Surface wrinkling, as a very common phenomenon in nature [1], e.g., wrinkles on the human face and the surface of dried fruits, has attracted wide attention due to its diverse applications, including thin film metrology [2], stretchable electronics [3], anticounterfeiting [4], structured templates [5] and so on [6,7]. The surface wrinkling system is usually a bilayer system, with a stiff film attached to a compliant substrate. Due to the modulus mismatch of the film and substrate, once the applied in-plane compressive stress $(\sigma)$ in the film exceeds the bilayer-determined critical wrinkling stress $\left(\sigma_{\mathrm{c}}\right)$, the balance of the system is broken, resulting in surface wrinkles [8].

$$
\sigma_{\mathrm{c}}=\frac{\bar{E}_{f}}{4}\left(\frac{3 \bar{E}_{s}}{\bar{E}_{f}}\right)^{2 / 3}
$$

Here, $\bar{E}_{f}$ is the plane strain modulus given by $E /\left(1-v^{2}\right), E$ is Young's modulus, and $v$ is Poisson's ratio [1,6-8]. The subscripts " $f$ " and " $s$ " refer to the film and substrate, respectively. Note that the above equation gives the required critical wrinkling stress of a thin stiff film/thick compliant substrate bilayer system.

So far, considerable efforts have been made to manipulate the wrinkled morphologies in film/substrate systems. Given the stress-relief mechanism, dynamic variation of a stress/strain field is a familiar strategy to tune the wrinkling patterns, which could always be implemented by introducing a functional film. For example, large-scale wrinkle patterns on polyaniline (PANI) film/polydimethylsiloxane (PDMS) substrate could be fabricated by a swelling-induced self-wrinkling mechanism, and reversible modulation between 
the wrinkled and de-wrinkled states could be achieved by the unique acid doping/base dedoping of PANI [9]. Additionally, the intrinsic redox states of PANI, coupled with the corresponding volume expansion/shrinkage, have also been made full use of to control the switch between the wrinkled and de-wrinkled states on PANI film [10]. Introducing azobenzene units into azopolymer films or introducing a photosensitive intermediate layer in the multilayer system could lead to a dynamic and reversible wrinkle evolution by visible light-induced photoisomerization of polymer films [4,11,12]. However, a way to dynamically regulate the stress/strain field and further dynamically regulate the wrinkle morphologies, independent of a functional film, is still a challenge.

On the other hand, as shown in the above equation, the softer the substrate, the smaller the required critical wrinkling stress, namely, the bilayer system with a softer substrate wrinkles more easily. PDMS is a commonly used soft substrate. However, compared with a soft solid, fluid is much softer so that it is favored to act as a soft substrate. Water, as a typical fluid and a special soft substrate, has been utilized to investigate the surface wrinkling of floating polymer film [2,13-19]. For example, dripping a water droplet on the floating polymer film could induce the appearance of radial wrinkles, owing to the capillary force exerted by the water droplet at the contact line of air-water-polymer film [20]. When a steel ball is used to impact the floating film, it compresses the film downward, and further generates radial stress in the radial direction and compressive stress in the circumferential direction. In order to alleviate this compressive stress, radial wrinkles are triggered, and a series of surface morphologies evolve [21]. Additionally, loading by poking with a needle on the floating film has a similar wrinkling behavior as the impact of a steel ball.

Although some work has been reported on the surface wrinkling of the floating film, it is very difficult to control surface wrinkles on the floating film dynamically and reversibly. Whether dripping a water droplet on the floating film, or a steel ball impacting the floating film, or poking it with a needle, the floating film could not achieve the dynamic and reversible evolution of surface wrinkles. Moreover, the impact of a steel ball and poking with a needle may cause plastic deformation of the floating film, and even destroy the floating film. Recently, a magnetic drive, as a flexible, convenient, and special driving method, has been widely employed in many systems, such as using a magnetic drive to remotely operate droplets [22], magnetized micropillar arrays to transport droplets [23], magnetic artificial cilia [24], multi-legged magnetic soft robots [25], magnetic bionic rose petals [26], a magnetic anisotropic brake $[27,28]$ and so on.

In this paper, we developed a simple, novel, yet robust strategy to regulate the surface wrinkles on a floating film dynamically and reversibly. Specifically, a magnetic droplet containing superparamagnetic $\mathrm{Fe}_{3} \mathrm{O}_{4}$ particles [29] was dripped on the floating PS film, and a neodymium magnet was placed directly below the magnetic droplet. At this moment, the magnetic droplet is subjected to a relatively large magnetic force and compresses the floating PS film, leading to radial stress in the radial direction, and further, compressive stress in the circumferential direction. Under the action of this compressive stress, radial wrinkles emerge on the floating PS film. With the up-down movement of the neodymium magnet, these radial wrinkles evolve dynamically and reversibly with no apparent hysteresis and irreversible plastic deformation of the film. With the decrease in distance between the magnetic droplet and neodymium magnet, the wrinkle number increased, and a wrinkle-fold transition could be clearly observed. Furthermore, the influence of floating film thickness, magnetic droplet size, and the coupling effect of multiple droplets on surface wrinkling has also been systematically studied on the floating film in an external magnetic field. In principle, our simple, versatile approach could be conveniently extended to other film/fluid systems for the controlled evolution of prescribed surface patterns with desirable properties and functionalities.

\section{Materials and Methods}

Polystyrene (PS) $\left(M_{w}=250,000 \mathrm{~g} / \mathrm{mol}\right.$, ACROS, Beijing, China), polyetherimide (PEI) (anhydrous, 99.9\%, HEOWNS, Tianjin, China), and tetrahydrofuran (anhydrous, 99.9\%, 
Aladdin, Shanghai, China) were used without further purification. Polytetrafluoroethylene (PTFE) (average particle size: $5 \mu \mathrm{m}$, HEOWNS, Tianjin, China) was used to wrap the magnetic droplet. Iron (III) chloride $\left(\mathrm{FeCl}_{3}\right)(98 \%$, Aladdin, Shanghai, China), Iron (II) sulfate heptahydrate $\left(\mathrm{FeSO}_{4} \cdot 7 \mathrm{H}_{2} \mathrm{O}\right)(98 \%$, Aladdin, Shanghai, China), and sodium hydroxide $(\mathrm{NaOH})(96 \%$, MACKLIN, Shanghai, China) were used to prepare ferroferric oxide $\left(\mathrm{Fe}_{3} \mathrm{O}_{4}\right)$. $\mathrm{FeCl}_{3}$ powders are black-brown crystals or flakes. They can absorb moisture in the air and lead to deliquescence, namely, it is easily soluble in water and has strong water absorption. In our experimental system, $\mathrm{FeCl}_{3}$ provides $\mathrm{Fe}^{3+}$ for the reaction in the process of preparing $\mathrm{Fe}_{3} \mathrm{O}_{4}$ particles by co-precipitation, and thereinto $\mathrm{Fe}^{3+}$ renders a reaction-required oxidizing property.

\subsection{Fabrication of Floating Polymer Films}

Firstly, PEI solution in absolute ethyl alcohol and PS solution in tetrahydrofuran were prepared, respectively. The concentration of PEI solution is fixed to be $3 \mathrm{wt} \%$, while the concentration of PS solution ranges from $2 \mathrm{wt} \%$ to $6 \mathrm{wt} \%$. Secondly, PEI solution and PS solution were sequentially spin-coated on the circular cover glass with a diameter of $2 \mathrm{~cm}$, to fabricate the planar PS/PEI bilayer films. Thereinto, the thickness of PS film can be controlled by varying the concentration of PS solution and spin-coating speed. The spin-coating speed ranges from $2000 \mathrm{rpm}$ to $5000 \mathrm{rpm}$. Then, the PS/PEI bilayer films were put on the circular cover glass into water. Since PEI is a water-soluble polymer, the PEI film close to the cover glass dissolved in the water quickly, finally causing the PS film to detach from the cover glass and float freely on the water surface.

\subsection{Fabrication of Magnetic Droplets}

Firstly, superparamagnetic $\mathrm{Fe}_{3} \mathrm{O}_{4}$ powders were prepared. Specifically, pre-weighed $\mathrm{FeCl}_{3}$ powders and $\mathrm{FeSO}_{4} \cdot 7 \mathrm{H}_{2} \mathrm{O}$ powders were sequentially poured into a beaker containing a proper amount of distilled water, to make the molar concentration ratio of $\mathrm{Fe}^{3+}: \mathrm{Fe}^{2+}$ to be 1:2. A strong mechanical stirring was performed, to form a uniform mixture solution, and then $0.4 \mathrm{~mol} / \mathrm{L}$ ammonia water was added dropwise until the $\mathrm{pH}$ value of the mixture solution was 10. Afterward, this mixture solution was transferred to a three-necked flask in the protection of nitrogen and was heated in a water bath at $80^{\circ} \mathrm{C}$ for $30 \mathrm{~min}$. During heating, black precipitation gradually appeared in the bottom of the three-necked flask and was kept for $30 \mathrm{~min}$, then it was oven-dried at $80^{\circ} \mathrm{C}$ for $6 \mathrm{~h}$ to get the superparamagnetic $\mathrm{Fe}_{3} \mathrm{O}_{4}$ powders.

Subsequently, the pre-prepared superparamagnetic $\mathrm{Fe}_{3} \mathrm{O}_{4}$ powders were ultrasonically dispersed in water uniformly, and magnetic droplets were obtained by transferring the trace aqueous solution using a pipette. Additionally, magnetic liquid marbles were also prepared, by transferring a $4 \mu \mathrm{L}$ magnetic droplet to the PTFE powders and shaking for a few seconds. Since PTFE powders are a kind of hydrophobic material, they are inclined to wrap the droplet, and finally, a magnetic liquid marble was formed.

\subsection{In-Situ Observation of Surface Morphologies on Floating PS Films under Magnetic Control}

A magnetic droplet was transferred onto the center of the floating PS film, and a neodymium magnet was placed directly below the magnetic droplet, making the magnetic droplet, the center of the floating PS film, and the neodymium magnet fall into a straight line in the vertical direction. Subsequently, the changes of surface morphologies on the floating PS films with the up-down movement of the neodymium magnet in the vertical direction were observed in situ.

\subsection{Characterization}

The dynamic reversible evolution of surface morphologies on the floating film was captured by a stereomicroscope (Nikon SMZ 745T, Tokyo, Japan). XL-30 scanning electron microscopy (Philips, Endorven, the Netherlands) was used to photograph the cross-section of the film, to characterize the film thickness. The adjustment of the distance between 
the magnetic droplet and the magnet is realized by a TYZD motion controller (Krater, Shenzhen, China).

\section{Results and Discussion}

A schematic diagram of the experimental setup is shown in Figure 1a. The whole experimental process was divided into three steps. In the first step, a PS film was transferred onto the water surface. In the action of surface tension that $\sigma$ exerted at the film boundary, the PS film spread out quickly and floated freely on the water surface like a flat sheet of paper (Figure 1c). In the second step, a $4 \mu \mathrm{L}$ magnetic droplet was placed in the center of the floating PS film by using a pipette. A certain number and length of surface wrinkles were obviously observed at and near the air-magnetic droplet-PS contact line within tens of milliseconds (Figure 1d). When the magnetic droplet was placed in the center of the floating PS film, the PS film was subjected to the gravity $G$ of the magnetic droplet and the capillary force $\gamma$ exerted by the magnetic droplet [13] (see Figure 1b). These two forces could induce radial stress in the radial direction of the PS film, which could stretch the PS film in the radial direction. At this moment, compressive stress generates around the circumferential direction of the PS film and the floating PS film presents an unstable state. As soon as this compressive stress exceeds the required critical stress for PS film wrinkling, the floating PS film would wrinkle, as shown in Figure $1 \mathrm{~d}$.

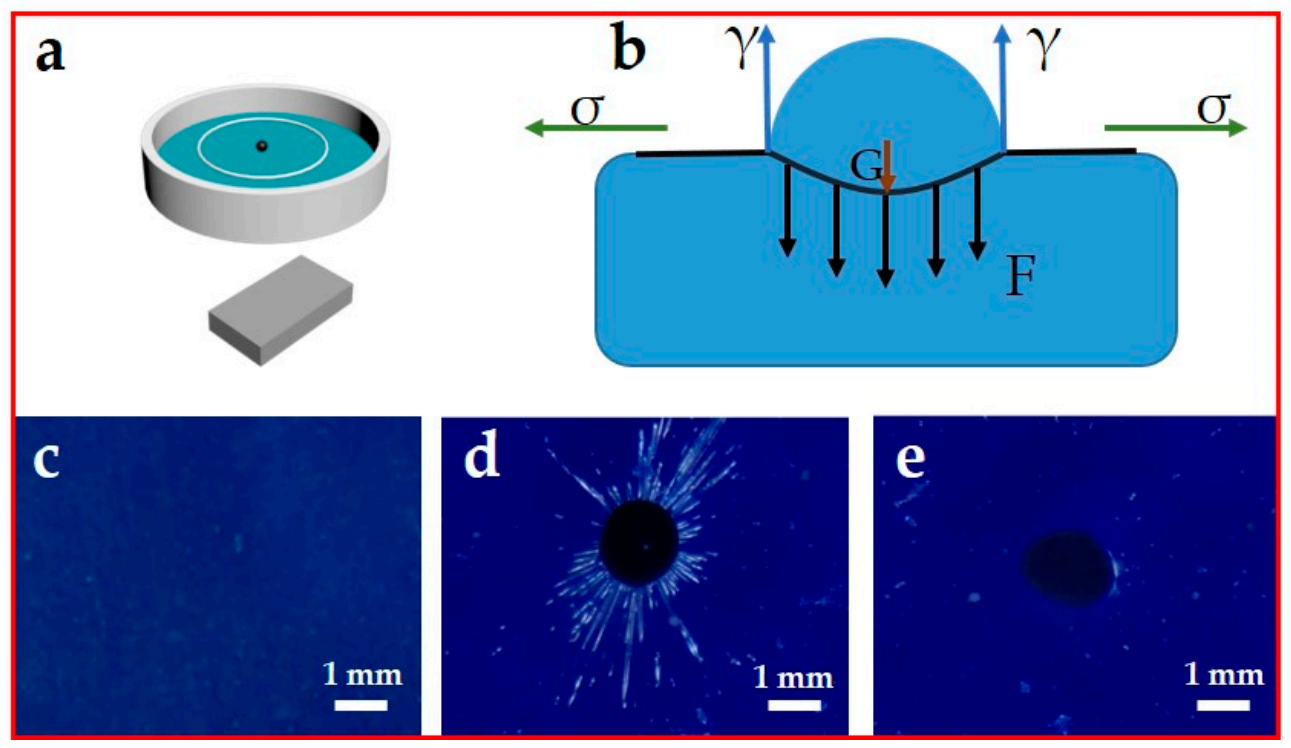

Figure 1. (a) Schematic diagram of the magnetically induced wrinkling of a floating PS film. (b) Forces that the floating PS film are subjected to when a magnetic droplet is dipped on the floating PS film: $\sigma$ is the surface tension of water, $\gamma$ is the capillary force exerted by the magnetic droplet, $G$ is the gravity of the magnetic droplet and F is magnetic force. (c) The floating PS film with nothing on it. (d) The floating PS film with a $4 \mu \mathrm{L}$ magnetic droplet on it. (e) The floating PS film with a $4 \mu \mathrm{L}$ magnetic droplet wrapped with PTFE powders on it. The thickness of PS film is $100 \mathrm{~nm}$ in (c-e).

Additionally, the case of a magnetic liquid marble, which was fabricated by wrapping a layer of hydrophobic PTFE powders outside the magnetic droplet, has also been studied. When a magnetic liquid marble containing the same volume of magnetic droplet as the one in Figure 1d was placed in the center of the floating PS film, the PS film remained flat and no obvious wrinkles were observed (Figure 1e). In this case, the floating PS film was only subjected to the gravity $\mathrm{G}$ of the magnetic liquid marble itself, but no capillary force was exerted by the magnetic liquid marble, since the magnetic liquid marble is similar to a soft solid, not a pure liquid droplet. Therefore, it follows that the reason why the floating PS film wrinkles in the case of the magnetic droplet is mainly from the action of capillary force exerted by the magnetic droplet, not its own gravity. Here, surface wrinkling on the 
floating PS film was consistent with the previously reported results, and is called "capillary wrinkling" [14].

In the third step, a neodymium magnet (Figure S1) was placed directly below the magnetic droplet, making the magnetic droplet, the center of the floating PS film, and the neodymium magnet fall into a straight line in the vertical direction. Hereinto the neodymium magnet is a tetragonal crystal composed of neodymium, iron, and boron, and is usually referred to directly as a $\mathrm{NdFeB}$ magnet. It possesses high saturation magnetization, high uniaxial anisotropy, and a high Curie temperature. Furthermore, it is the most magnetic permanent magnet in mainstream use and is also the most widely used rare earth permanent magnet material, since neodymium is a rare earth element. At this moment, the PS film is subjected to not only the gravity $\mathrm{G}$ of the magnetic droplet itself and the capillary force $\gamma$ exerted by the magnetic droplet, but also the magnetic force $\mathrm{F}$ from the attraction of the neodymium magnet to the magnetic droplet (Figure 1b). As mentioned above, the gravity of the magnetic droplet itself has no effect on the surface wrinkling of the floating PS film. Furthermore, compared with the scenario of no neodymium magnet, the addition of neodymium magnet makes the floating PS film wrinkling more obvious, and the scope of surface wrinkles is larger, almost spreading over the whole PS film (Figure 2, Video S1). Therefore, it can be attested that the contribution of magnetic force from the attraction of the neodymium magnet to the magnetic droplet on the PS film wrinkling is far greater than the capillary force exerted by the magnetic droplet. In other words, although magnetic force from the attraction of the neodymium magnet to the magnetic droplet, combined with the capillary force exerted by the magnetic droplet, collectively induces radial stress in the radial direction, and further, compressive stress in the circumferential direction, the magnetic force from the attraction of the neodymium magnet to the magnetic droplet is still the main factor to trigger surface wrinkles on the floating PS film in our current system.

The magnetic force from the attraction of the neodymium magnet to the magnetic droplet could be easily tailored by varying the distance between the neodymium magnet and the PS film using a motion controller. The changes of surface morphologies on the floating PS films with the up-down movement of the neodymium magnet in the vertical direction have been observed in situ under a stereo optical microscope. When the neodymium magnet gradually approached the magnetic droplet, e.g., from $19 \mathrm{~mm}$ to $9.34 \mathrm{~mm}$ away from the magnetic droplet, an obvious circular halo appeared on the PS film around the magnetic droplet, and the number of surface wrinkles increased from 156 to 367 (Figure 2a,b,h). With the approach of the neodymium magnet to the magnetic droplet, namely, with the decrease in the distance between the magnet and the magnetic droplet, the magnetic force exerted on the PS film increased so that the PS film was stretched inward in the radial direction and a displacement of the PS film center in the vertical direction, called indentation depth, is generated at this time. This indentation depth results in the visual circular halo effect. Meanwhile, with the increase in magnetic force, the radial stress exerted on the film in the radial direction increased and further, the induced compressive stress in the circumferential direction synchronously increased, finally leading to a decrease in the wrinkle wavelength, and in turn, an increase in the wrinkle number [21]. Further increasing the distance between the magnet and the magnetic droplet to $7.457 \mathrm{~mm}$, the circular halo expands outward, a small number of folds come out, and the wrinkle number decreases to 345 (Figure 2c,h). The increase in magnetic force gives rise to a larger indentation depth, leading to a larger circular halo range. Additionally, the greater the magnetic force, the greater the radial stress exerted on the film in the radial direction and the induced compressive stress in the circumferential direction. When this compressive stress exceeds a critical value, the bifurcation of surface instabilities of the film happens, showing a wrinkle to fold transition to minimize the total system energy here [19]. The appearance of film folds alleviates partial stresses so that the wrinkle number decreases in comparison to that in Figure 2b. When the neodymium magnet further approaches the magnetic droplet to $6 \mathrm{~mm}$, this phenomenon is more obvious as shown in Figure 2d. Typically, the range of the circular halo is larger, the depth and number of film folds further increase, and the 
wrinkle number continues to decrease to 161 (Figure 2d,h). Subsequently, the magnet is slowly moved down to its initial location, i.e., $19 \mathrm{~mm}$ away from the magnetic droplet. It can be seen that the circular halo gradually shrinks until it disappears, film folds gradually become less obvious until they disappear, and the variation trend of wrinkle number almost coincides with the case of magnet movement upward (Figure 2e-h). In simple terms, in the absence of magnetic field surface wrinkles on the floating PS film are only related to the capillary force of the magnetic droplet. At this time, the capillary force is relatively small, and the floating PS film is in a low compression state, resulting in a small range of radial wrinkles with a relatively small wrinkle number. However, when the magnetic force is introduced, the floating PS film is in a relatively high compression state, and the wrinkle number gradually increases with the increase in magnetic force. When further increasing the magnetic force, the compression process continues, the wrinkle amplitude increases, and folds in a state of high local curvature will appear. Eventually, the amplitude of several wrinkles increases, while others decay, and all distortions are concentrated in a few narrow areas on the floating PS film surface. The appearance of film folds made the wrinkle number sharply decrease, i.e., a maximum value of the wrinkle number appears in Figure $2 \mathrm{~h}$.

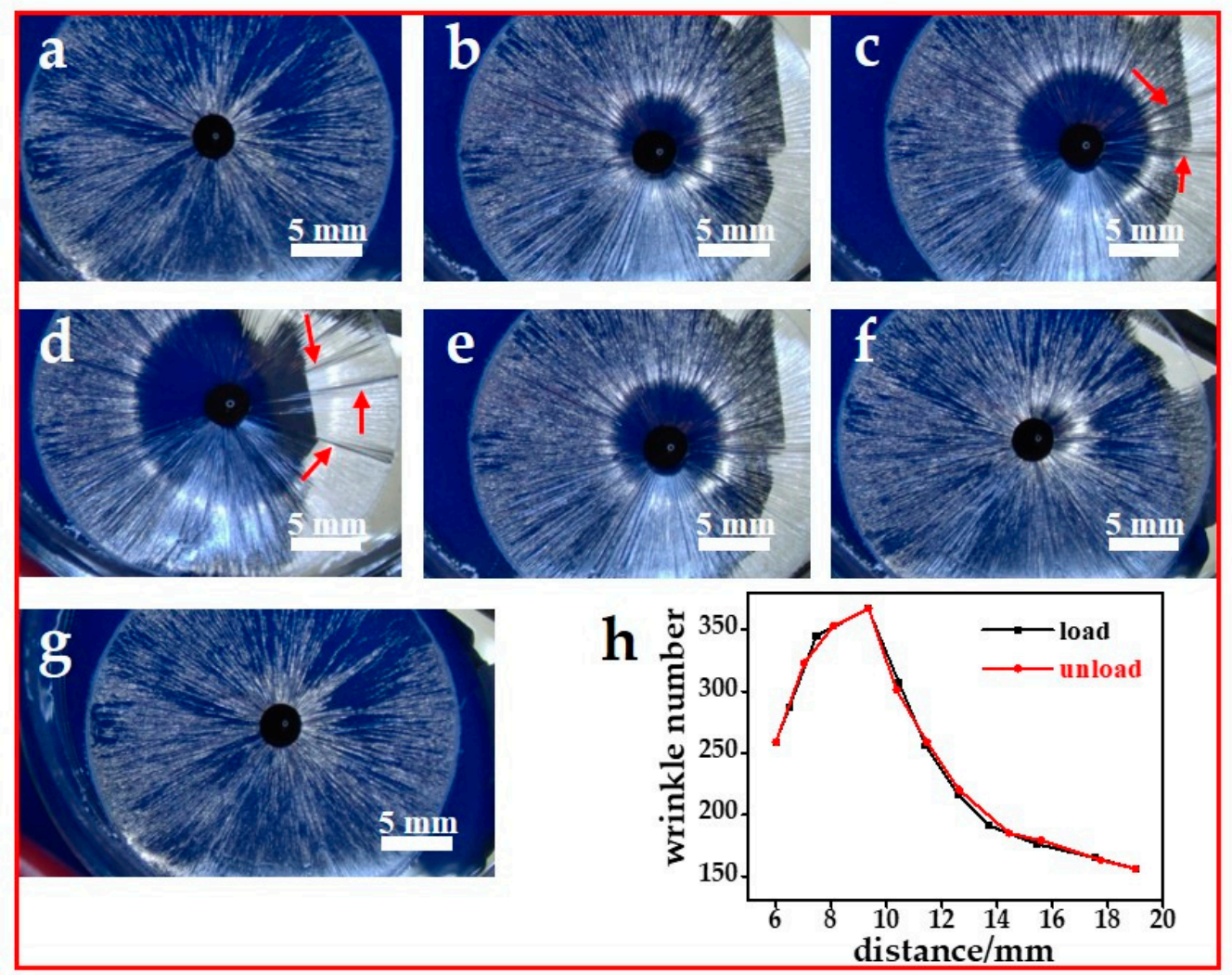

Figure 2. Optical microscopy images of surface morphologies of the floating PS film with a $4 \mu \mathrm{L}$ magnetic droplet on it. The PS film is $100 \mathrm{~nm}$ thick, and the distance between the magnet and the magnetic droplet is (a) $19 \mathrm{~mm}$, (b) $9.336 \mathrm{~mm}$, (c) $7.457 \mathrm{~mm}$, (d) $6 \mathrm{~mm}$, (e) $8.524 \mathrm{~mm}$, (f) $11.409 \mathrm{~mm}$, and (g) $19 \mathrm{~mm}$, respectively. (h) The relationship of the wrinkle number versus the distance between the magnet and the magnetic droplet in the dynamic and reversible process of loading and unloading the magnetic field.

Here it is emphasized that the magnetic force dominates the surface wrinkling and folding behaviors of the PS floating film in our system. However, the magnetic force in our system was influenced by many factors, such as the content of magnetic $\mathrm{Fe}_{3} \mathrm{O}_{4}$ particles in the magnetic droplet, magnetic droplet size, floating polymer film, fluid substrate water, the distance between the magnet and the magnetic droplet, and so on, which made 
the measurement of magnetic force complicated and difficult. In fact, the uncertainty of the magnetic force did not affect control over the surface morphologies of the floating polymer film. Upon fixing other factors, e.g., the content of magnetic $\mathrm{Fe}_{3} \mathrm{O}_{4}$ particles in the magnetic droplet, magnetic droplet size, floating polymer film thickness, and fluid substrate water, the magnetic force was only related to the distance between the magnet and the magnetic droplet. Thus far, it has been fully demonstrated that the evolution of surface morphologies on the floating polymer film can be dynamically and reversibly regulated by controlling the external magnetic field, i.e., the up-down movement of the magnet and the distance between the magnet and the magnetic droplet. As is different from the previously reported theoretical simulation and analysis [30], we have successfully achieved the dynamic reversible evolution of wrinkles and folds on floating polymer films under magnetic control in the experiments for the first time, to the best of our knowledge. Therefore, it is reasonable to say that this work is at least experimentally novel. Additionally, it is worthy to note that the dynamic and reversible evolution of surface wrinkles in our system causes no damage to the polymer film, and no hysteretic effect on the evolution process, which is fundamentally different from a steel ball impacting the floating film, and dripping a water droplet on the floating film [21,31].

The influence of film thickness on the surface morphology evolution process of the floating PS film has been explored, when the neodymium magnet is about $7.5 \mathrm{~mm}$ away from the magnetic droplet and the volume of the magnetic droplet is $4 \mu \mathrm{L}$. It can be seen that, with the increase in PS film thickness from $100 \mathrm{~nm}, 150 \mathrm{~nm}, 200 \mathrm{~nm}, 250 \mathrm{~nm}$ to $300 \mathrm{~nm}$, the circular halo around the magnetic droplet becomes less obvious until it disappears, and the wrinkle number in the PS film becomes smaller and smaller (Figure 3a-e). Specifically, the wrinkle number in the PS film with film thickness from $100 \mathrm{~nm}, 150 \mathrm{~nm}, 200 \mathrm{~nm}$, $250 \mathrm{~nm}$ to $300 \mathrm{~nm}$ is $367,175,85,54$, and 20, respectively, as shown in Figure 3f. It is well known that the thicker the polymer film, the more difficult it is to bend, namely, the thicker polymer film possesses greater intrinsic bending rigidity. Therefore, in the case of the same distance between the magnet and the magnetic droplet and the same volume of the magnetic droplet, the thicker PS film is subjected to lesser radial stress in the radial direction and lesser compressive stress in the circumferential direction. The thicker PS film with lesser radial stress in the radial direction is more difficult to stretch with the magnetic force in the vertical direction, so that the indentation depth reduces or even disappears, and furthermore, the circular halo shrinks or even disappears. On the other hand, the thicker PS film with lesser compressive stress in the circumferential direction would present a larger wrinkle wavelength, and a smaller wrinkle number. According to the data of wrinkle number $\mathrm{N}$ and film thickness $\mathrm{h}$ presented in Figure $3 \mathrm{f}$, the logarithm of wrinkle number $\lg N$ versus the logarithm of film thickness lgh has been re-plotted and fitted in Figure S2. The logarithm of wrinkle number $\operatorname{lgN}$ decreases linearly with the logarithm of film thickness lgh, and the fitted slope is -2.51 . It can be deduced that $\mathrm{N} \sim \mathrm{k} \cdot \mathrm{h}^{-2.51}$ could be satisfied in our system. In the capillary wrinkling of the floating PS film by dripping a water droplet, the relationship of the wrinkle number $\mathrm{N}$ with the film thickness $\mathrm{h}$ gives an equation $\mathrm{N} \sim \mathrm{a}^{1 / 2} \mathrm{~h}^{-3 / 4}$, in which a is the radius of the droplet [2,14]. Different laws between $\mathrm{N} \sim \mathrm{k} \cdot \mathrm{h}^{-2.51}$ in our system and $\mathrm{N} \sim \mathrm{a}^{1 / 2} \mathrm{~h}^{-3 / 4}$ in the capillary wrinkling basically originate from the slight differences of experimental systems. For example, a magnetic droplet is applied in our system while a pure water droplet is applied in the capillary wrinkling, an external magnetic field is imposed in our system while there is no magnetic interference in the capillary wrinkling. Even so, the variation trend of the wrinkle number $\mathrm{N}$ with the film thickness $\mathrm{h}$ in our current system is still consistent with the reported results in the capillary wrinkling. Certainly, the technique and experimental results presented here could be made full use of when determining the parameters of floating film, such as film thickness. A relationship of the wrinkle number to film thickness has been captured when other parameters, e.g., magnetic droplet size and the distance between the magnet and the magnetic droplet, are fixed. Under the same conditions, as long as the wrinkle number is known, the film thickness could be simply deduced according to this captured relationship 
of wrinkle number to film thickness. In other words, we provide an innovative method for measuring the film thickness.

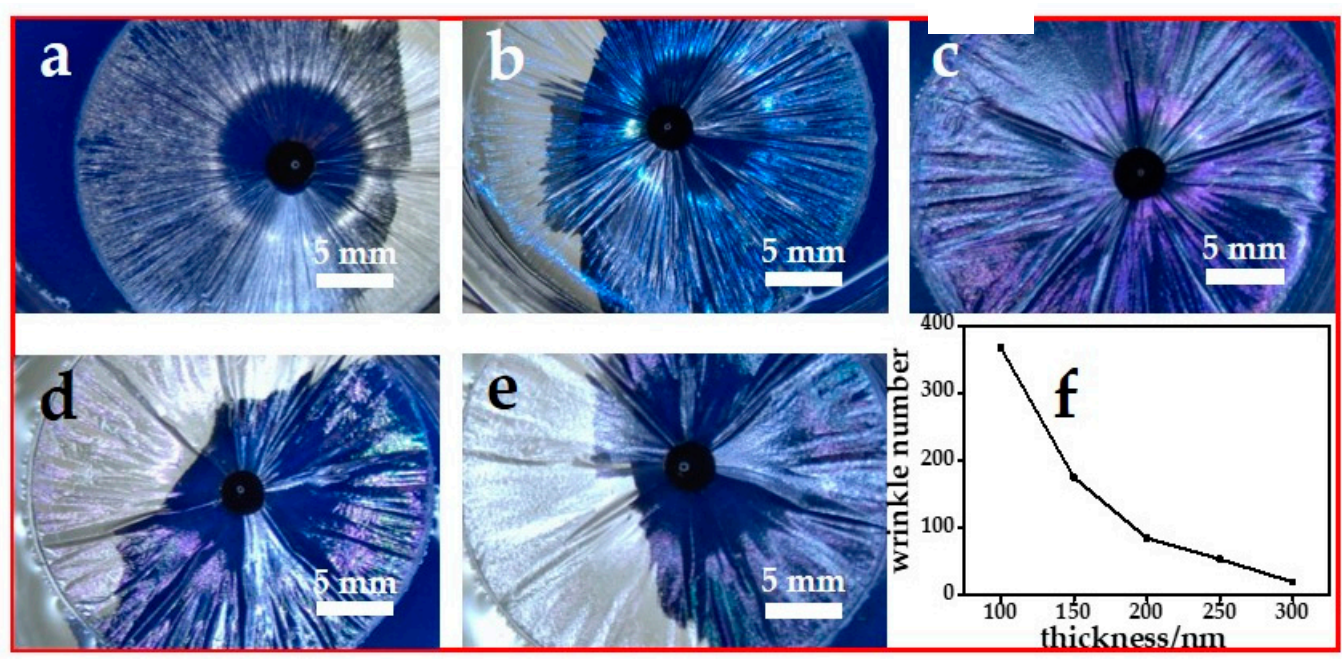

Figure 3. Optical microscopy images of surface morphologies of the floating PS film with (a) $100 \mathrm{~nm}$, (b) $150 \mathrm{~nm}$, (c) $200 \mathrm{~nm}$, (d) $250 \mathrm{~nm}$, (e) $300 \mathrm{~nm}$ thickness, when a $4 \mu \mathrm{L}$ magnetic droplet is dripped on the PS film and the magnet is $7.5 \mathrm{~mm}$ away from the magnetic droplet. (f) The relationship of the number of wrinkles to the film thickness.

The influence of magnetic droplet volume on the surface morphology evolution process of the floating PS film has also been explored when the neodymium magnet is about $7.5 \mathrm{~mm}$ away from the magnetic droplet and the PS film thickness is $100 \mathrm{~nm}$. With the increase in magnetic droplet volume from $2 \mu \mathrm{L}, 4 \mu \mathrm{L}, 6 \mu \mathrm{L}$ to $8 \mu \mathrm{L}$, the circular halo expanded rapidly, a wrinkle-fold transition occurred, and the wrinkle number first increased and then decreased (Figure 4). The larger the magnetic droplet, the stronger the attraction of the magnet to the magnetic droplet, and the greater the magnetic force exerted on the PS film. Therefore, in the case of the same distance between the magnet and the magnetic droplet and the same film thickness, a PS film with a larger magnetic droplet was subjected to greater radial stress in the radial direction and greater compressive stress in the circumferential direction. A PS film with greater radial stress in the radial direction was stretched by the magnetic force in the vertical direction more easily, so that the indentation depth increased, and furthermore, the circular halo expanded rapidly. On the other hand, a PS film with greater compressive stress in the circumferential direction would present a smaller wrinkle wavelength and a larger wrinkle number (Figure 4b-d). As above mentioned, when this induced compressive stress in the circumferential direction exceeded a critical value, the bifurcation of surface instabilities on the PS film happened, namely, a wrinkle-fold transition occurs in our current system (Figure 4e). Figure $4 \mathrm{f}$ shows that before the film folds, the wrinkle number increased constantly with the increase in the magnetic droplet volume, which is again consistent with the predicted tendency of the above equation $\mathrm{N} \sim \mathrm{a}^{1 / 2} \mathrm{~h}^{-3 / 4}$ in the capillary wrinkling of the floating PS film dripped a water droplet $[14,19]$. Further increasing this induced compressive stress, surface wrinkles gradually attenuated, and finally, only 9 folds remained (Figure $4 \mathrm{e}$ ). In simple terms, as the magnetic droplet size increasingly increased, the magnetic force gradually increased. When the magnetic force increased to a critical value, a similar phenomenon to Figure $2 \mathrm{~h}$ appeared in Figure $4 \mathrm{f}$ due to the bifurcation of surface instabilities of the floating polymer film, i.e., a wrinkle-to-fold transition to minimize the total system energy. Therefore, the plot in Figure $4 \mathrm{f}$ also has a maximum. 


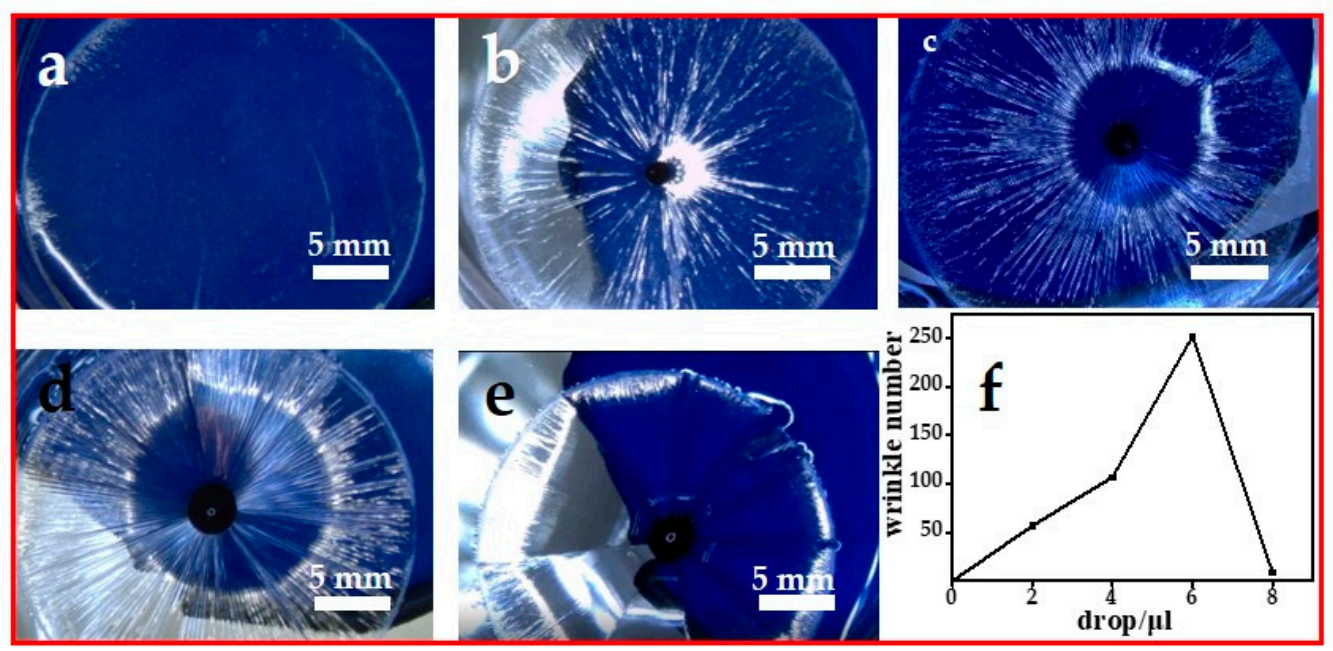

Figure 4. Optical microscopy images of surface morphologies on the floating PS film (a) without a magnetic droplet and with a (b) $2 \mu \mathrm{L}$, (c) $4 \mu \mathrm{L}$, (d) $6 \mu \mathrm{L}$, (e) $8 \mu \mathrm{L}$ magnetic droplet on it, when the PS film is $100 \mathrm{~nm}$ thick, and the magnet is $7.5 \mathrm{~mm}$ away from the magnetic droplets. (f) The relationship of wrinkle number versus magnetic droplet size, thereinto the experimental point that the $8 \mu \mathrm{L}$ magnetic droplet corresponds to is the fold number, not the wrinkle number.

Furthermore, an electromagnet instead of a permanent magnet has been used in attempts to investigate the dynamic evolution of surface morphologies of the floating polymer film. In the case of the electromagnet, the magnetic force could be simply regulated with the current through the coil. Specifically, a Keithley K2400 digital source meter (Keithley, Cleveland, America) was utilized to impose a constant voltage $12 \mathrm{~V}$ to the electromagnet (working voltage $12 \mathrm{~V}$ ) and the corresponding current ranged from $-1.05 \mathrm{~A}$ to $+1.05 \mathrm{~A}$. The experimental setup is shown in Figure S3, and the dynamic evolutions of surface morphologies of the floating PS film with the current or the magnetic droplet size in the case of the electromagnet have been shown in Figures S4 and S5. It can be seen that the number and range of radial wrinkles on the floating PS film increasingly increased with the current or the magnetic droplet size. Whether the current or the magnetic droplet size increased, the magnetic force the floating PS film is subjected to increased. Finally, the greater magnetic force resulted in the larger wrinkle number and the larger wrinkling area, which is similar to the case of the permanent magnet. The dynamic evolution of surface morphologies of the floating PS film in the case of the electromagnet was not as obvious as that of the permanent magnet. It is believed that the optimum conditions have not been captured, and further exploration is still underway (Video S2).

In addition to the case of a single magnetic droplet dripped on the floating PS film, here the influence of multiple magnetic droplets on the surface morphologies of the floating PS film has also been systematically studied. Three $2 \mu \mathrm{L}$ magnetic droplets were simultaneously placed on the PS film of $100 \mathrm{~nm}$ thickness, and they almost formed an equilateral triangle. When the magnet was placed directly below the center of the equilateral triangle composed of three magnetic droplets, surface wrinkles with the same wrinkle number surrounding every magnetic droplet were triggered, mainly due to the magnetic force exerted on the film, and there were also interconnected wrinkles or folds between the adjacent two magnetic droplets to form a triangular pattern (Figure 5a). Further increasing the PS film thickness to $200 \mathrm{~nm}$ with the other conditions invariable, similar surface morphologies as those in Figure 5a appeared on the PS film, except the wrinkle number around every magnetic droplet was smaller than that in Figure 5a. Moreover, different volumes of magnetic droplets, e.g., $2 \mu \mathrm{L}, 4 \mu \mathrm{L}$, and $6 \mu \mathrm{L}$, were placed on the PS film of $100 \mathrm{~nm}$ thick, according to the same arrangement form as in Figure 5a,c. Because different volumes of magnetic droplets were subjected to different degrees of magnetic attraction, and further exert different magnetic forces, three sets of surface wrinkles with different 
wrinkle numbers were generated on the PS film. It can also be seen that the $6 \mu \mathrm{L}$ magnetic droplet exerted the largest magnetic force on the PS film, so that it could couple with the $2 \mu \mathrm{L}$ and $4 \mu \mathrm{L}$ magnetic droplets to make the surface wrinkles penetrate each other in the horizontal direction, as shown in Figure 5b. Further increasing the PS film thickness to $200 \mathrm{~nm}$, with the other conditions invariable, similar surface morphologies as those in Figure 5b appeared on the PS film, except the wrinkle number around every magnetic droplet was smaller than that in Figure $5 \mathrm{~b}$. So far it can be confirmed that novel surface wrinkle patterns can easily be achieved by the simple coupling of multiple magnetic droplets on the floating PS film, under the external magnetic field.

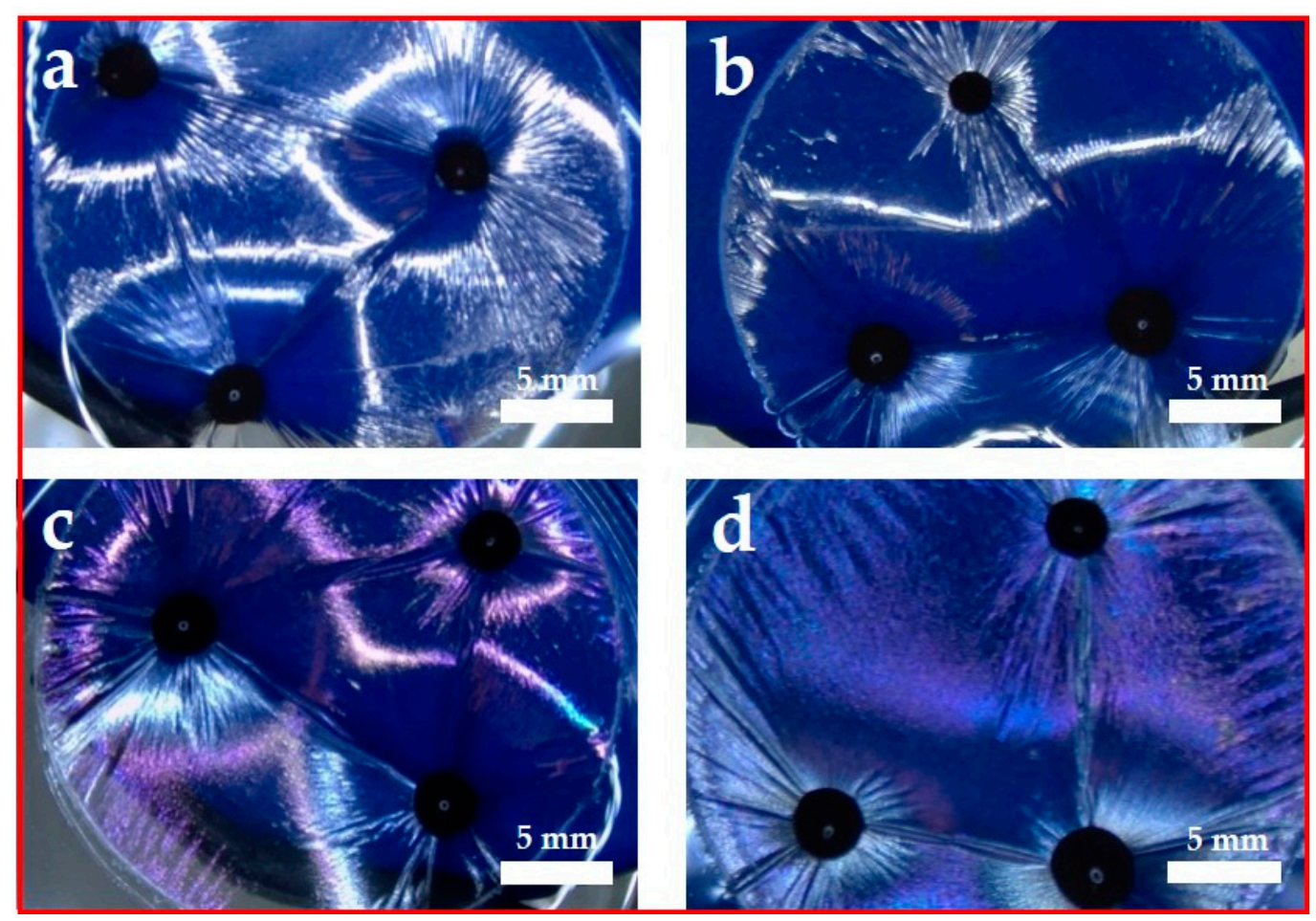

Figure 5. Optical microscopy images of surface morphologies on the floating PS film with three magnetic droplets on it. Three $4 \mu \mathrm{L}$ magnetic droplets were placed on a PS film of (a) $100 \mathrm{~nm}$ and (c) $200 \mathrm{~nm}$ thick. Three magnetic droplets with the volumes of $2 \mu \mathrm{L}, 4 \mu \mathrm{L}$, and $6 \mu \mathrm{L}$ are placed on a PS film of (b) $100 \mathrm{~nm}$ and (d) $200 \mathrm{~nm}$ thick.

\section{Conclusions}

In summary, dynamic and reversible wrinkles on the floating PS film under the magnetic control have been controllably fabricated for the first time. This was achieved by dripping a magnetic droplet on the floating PS film and placing a neodymium magnet directly below the magnetic droplet. Surface morphologies of the floating PS film, including the wrinkle number and the mutual transition between wrinkles and folds, can thus be modulated in a predictable way, using experimental parameters such as the distance between the magnetic droplet and the neodymium magnet, PS film thickness, and magnetic droplet volume. The closer the neodymium magnet is to the magnetic droplet, or the thinner the PS film is, or the larger the magnetic droplet, the larger the wrinkle number is, and surface wrinkles transform to folds more easily. Additionally, novel surface wrinkle patterns can be easily realized by the simple coupling of multiple magnetic droplets on the same floating PS film, under the external magnetic field. Therefore, this simple, universal yet robust floating film wrinkling technique, based on magnetic force, not only sheds new insights into the floating film wrinkling mechanism, but also opens up enormous possibilities to create unprecedented surface patterns with multifunctional capability. 
Supplementary Materials: The following are available online at https: / www.mdpi.com/article/ 10.3390/coatings11050494/s1, Figure S1: The relationship between magnetic flux density of the used permanent magnet and distance away from its surface, Figure S2: The plot of the logarithm of wrinkle number $\operatorname{lgN}$ versus the logarithm of film thickness lgh, Figure S3: The experimental setup of magnetic control over the surface morphologies of the floating polymer films through an electromagnet, Figure S4: Optical microscopy images of surface morphologies of the floating PS film with a $4 \mu \mathrm{L}$ magnetic droplet on it in the case of the electromagnet. PS film is $100 \mathrm{~nm}$ thick, and the current is (a) $0.2 \mathrm{~A}$, (b) $0.4 \mathrm{~A}$, (c) $0.6 \mathrm{~A}$, (d) $0.8 \mathrm{~A}$, (e) $1.0 \mathrm{~A}$, respectively. (f) The relationship of wrinkle number versus the current in the dynamic process of loading magnetic field, Figure S5: Optical microscopy images of surface morphologies on the floating PS film with a (a) $2 \mu \mathrm{L}$, (b) $6 \mu \mathrm{L}$, (c) $8 \mu \mathrm{L}$, (d) $10 \mu \mathrm{L}$ magnetic droplet on it, when the PS film is $100 \mathrm{~nm}$ thick and the current is $1.0 \mathrm{~A}$. (e) The relationship of wrinkle number versus magnetic droplet size, Video S1: Dynamic evolution of surface morphologies of the floating PS film with the change of distance between the magnet and the magnetic droplet, Video S2: Dynamic evolution of surface morphologies of the floating PS film with the change of current in the case of the electromagnet.

Author Contributions: Conceptualization, C.L.; methodology, J.W., L.L. and E.L.; software, J.W., L.L. and E.L.; validation, C.J., X.H., J.W., L.L., E.L. and C.L.; investigation, C.J.; resources, C.L.; writing-original draft preparation, C.J.; writing-review and editing, X.H. and C.L.; project administration, C.L.; funding acquisition, X.H., J.W. and C.L. All authors have read and agreed to the published version of the manuscript.

Funding: This research was funded by the National Natural Science Foundation of China (No. 21875160), the Natural Science Foundation of Tianjin (No. 20JCQNJC00870), and the Scientific Research Project of Tianjin Municipal Education Commission (No. 2020KJ054).

Institutional Review Board Statement: Not applicable.

Informed Consent Statement: Not applicable.

Data Availability Statement: Not applicable.

Acknowledgments: The authors acknowledge the financial support from the National Natural Science Foundation of China (No. 21875160), the Natural Science Foundation of Tianjin (No. 20JCQNJC00870), and the Scientific Research Project of Tianjin Municipal Education Commission (No. 2020KJ054).

Conflicts of Interest: The authors declare no competing financial interest.

\section{References}

1. Genzer, J.; Groenewold, J. Soft matter with hard skin: From skin wrinkles to templating and material characterization. Soft Matter 2006, 2, 310-323. [CrossRef] [PubMed]

2. Chang, J.; Toga, K.B.; Paulsen, J.D.; Menon, N.; Russell, T.P. Thickness dependence of the Young's modulus of polymer thin films. Macromolecules 2018, 51, 6764-6770. [CrossRef]

3. Yu, S.; Li, L.; Wang, J.; Liu, E.; Zhao, J.; Xu, F.; Cao, Y.; Lu, C. Light-boosting highly sensitive pressure sensors based on bioinspired multiscale surface structures. Adv. Funct. Mater. 2020, 30, 1907091. [CrossRef]

4. Zong, C.; Zhao, Y.; Ji, H.; Han, X.; Xie, J.; Wang, J.; Cao, Y.; Jiang, S.; Lu, C. Tuning and erasing surface wrinkles by reversible visible-light-induced photoisomerization. Angew. Chem. Int. Ed. 2016, 55, 3931-3935. [CrossRef] [PubMed]

5. Zong, C.; Hu, M.; Azhar, U.; Chen, X.; Zhang, Y.; Zhang, S.; Lu, C. Smart copolymer-functionalized flexible surfaces with photoswitchable wettability: From superhydrophobicity with "rose petal" effect to superhydrophilicity. ACS Appl. Mater. Interfaces 2019, 11, 25436-25444. [CrossRef]

6. Chung, J.Y.; Nolte, A.J.; Stafford, C.M. Surface wrinkling: A versatile platform for measuring thin-film properties. Adv. Mater. 2011, 23, 349-368. [CrossRef] [PubMed]

7. Howarter, J.A.; Stafford, C.M. Instabilities as a measurement tool for soft materials. Soft Matter 2010, 6, 5661-5666. [CrossRef]

8. Bowden, N.; Brittain, S.; Evans, A.G.; Hutchinson, J.W.; Whitesides, G.M. Spontaneous formation of ordered structures in thin films of metals supported on an elastomeric polymer. Nature 1998, 393, 146-149. [CrossRef]

9. Xie, J.; Han, X.; Zong, C.; Ji, H.; Lu, C. Large-area patterning of polyaniline film based on in situ self-wrinkling and its reversible doping/dedoping tunability. Macromolecules 2015, 48, 663-671. [CrossRef]

10. Xie, J.; Zong, C.; Han, X.; Ji, H.; Wang, J.; Yang, X.; Lu, C. Redox-switchable surface wrinkling on polyaniline film. Macromol. Rapid Commun. 2016, 37, 637-642. [CrossRef]

11. Zong, C.; Azhar, U.; Zhou, C.; Wang, J.; Zhang, L.; Cao, Y.; Zhang, S.; Jiang, S.; Lu, C. Photocontrollable wrinkle morphology evolution on azo-based multilayers for hierarchical surface micropatterns fabrication. Langmuir 2019, 35, 2601-2609. [CrossRef] [PubMed] 
12. Wang, J.; Zheng, Y.; Li, L.; Liu, E.; Zong, C.; Zhao, J.; Xie, J.; Xu, F.; König, T.A.F.; Cao, Y.; et al. All-optical reversible azo-based wrinkling patterns with high aspect ratio and polarization-independent orientation for light-responsive soft photonics. ACS Appl. Mater. Interfaces 2019, 11, 25595-25604. [CrossRef] [PubMed]

13. Toga, K.B.; Huang, J.; Cunningham, K.; Russell, T.P.; Menon, N. A drop on a floating sheet: Boundary conditions, topography and formation of wrinkles. Soft Matter 2013, 9, 8289-8296. [CrossRef]

14. Huang, J.; Juszkiewicz, M.; de Jeu, W.H.; Cerda, E.; Emrick, T.; Menon, N.; Russell, T.P. Capillary wrinkling of floating thin polymer films. Science 2007, 317, 650-653. [CrossRef]

15. Paulsen, J.D.; Hohlfeld, E.; King, H.; Huang, J.; Qiu, Z.; Russell, T.P.; Menon, N.; Davidovitch, D.; Vella, B. Curvature-induced stiffness and the spatial variation of wavelength in wrinkled sheets. Proc. Natl. Acad. Sci. USA 2016, 113, 1144-1149. [CrossRef]

16. Oshri, O.; Brau, F.; Diamant, H. Wrinkles and folds in a fluid-supported sheet of finite size. Phys. Rev. E 2015, 91, 052408. [CrossRef]

17. Diamant, H.; Witten, T.A. Shape and symmetry of a fluid-supported elastic sheet. Phys. Rev. E 2013, 88, 012401. [CrossRef]

18. Huang, J.; Davidovitch, B.; Santangelo, C.D.; Russell, T.P.; Menon, N. Smooth cascade of wrinkles at the edge of a floating elastic film. Phys. Rev. Lett. 2010, 105, 038302. [CrossRef] [PubMed]

19. Pocivavsek, L.; Dellsy, R.; Kern, A.; Johnson, S.; Lin, B.; Lee, K.Y.C.; Cerda, E. Stress and fold localization in thin elastic membranes. Science 2008, 320, 912-916. [CrossRef]

20. Piñeirua, M.; Tanaka, N.; Roman, B.; Bico, J. Capillary buckling of a floating annulus. Soft Matter 2013, 9, 10985-10992. [CrossRef]

21. Box, F.; O'Kiely, D.; Kodio, O.; Inizan, M.; Castrejon-Pita, A.A.; Vella, D. Dynamics of wrinkling in ultrathin elastic sheets. Proc. Natl. Acad. Sci. USA 2019, 116, 20875-20880. [CrossRef] [PubMed]

22. Kim, J.H.; Kang, S.M.; Lee, B.J.; Ko, H.; Bae, W.-G.; Suh, K.Y.; Kwak, M.K.; Jeong, H.E. Remote manipulation of droplets on a flexible magnetically responsive film. Sci. Rep. 2015, 5, 17843. [CrossRef]

23. Lin, Y.; Hu, Z.; Zhang, M.; Xu, T.; Feng, S.; Jiang, L.; Zheng, Y. Magnetically induced low adhesive direction of nano/micropillar arrays for microdroplet transport. Adv. Funct. Mater. 2018, 28, 1800163. [CrossRef]

24. Timonen, J.V.I.; Johans, C.; Kontturi, K.; Walther, A.; Ikkala, O.; Ras, R.H.A. A facile template-free approach to magnetodriven, multifunctional artificial cilia. ACS Appl. Mater. Interfaces 2010, 2, 2226-2230. [CrossRef]

25. Lu, H.; Zhang, M.; Yang, Y.; Huang, Q.; Fukuda, T.; Wang, Z.; Shen, Y. A bioinspired multilegged soft millirobot that functions in both dry and wet conditions. Nat. Commun. 2018, 9, 3944. [CrossRef] [PubMed]

26. Gao, W.; Wang, L.; Wang, X.; Liu, H. Magnetic driving flowerlike soft platform: Biomimetic fabrication and external regulation. ACS Appl. Mater. Interfaces 2016, 8, 14182-14189. [CrossRef]

27. Kim, J.; Chung, S.E.; Choi, S.-E.; Lee, H.; Kim, J.; Kwon, S. Programming magnetic anisotropy in polymeric microactuators. Nat. Mater. 2011, 10, 747-752. [CrossRef] [PubMed]

28. Mishra, S.R.; Dickey, M.D.; Velev, O.D.; Tracy, J.B. Selective and directional actuation of elastomer films using chained magnetic nanoparticles. Nanoscale 2016, 8, 1309-1313. [CrossRef]

29. Shen, Y.F.; Tang, J.; Nie, Z.H.; Wang, Y.D.; Ren, Y.; Zuo, L. Preparation and application of magnetic $\mathrm{Fe}_{3} \mathrm{O}_{4}$ nanoparticles for wastewater purification. Sep. Purif. Technol. 2009, 68, 312-319. [CrossRef]

30. Anjos, P.H.A.; Carvalho, G.D.; Lira, S.A.; Miranda, J.A. Wrinkling and folding patterns in a confined ferrofluid droplet with an elastic interface. Phys. Rev. E. 2019, 99, 022608. [CrossRef] [PubMed]

31. O'Kiely, D.; Box, F.; Kodio, O.; Whiteley, J.; Vella, D. Impact on floating thin elastic sheets: A mathematical model. Phys. Rev. Fluids 2020, 5, 014003. [CrossRef] 